\title{
Experiência docente em saúde indígena: reflexões e movimentos
}

\author{
Teaching experience in indigenous health: reflections and \\ movements
}

\author{
Elenita Sureke Abilio', Maria José de Jesus Alves Cordeiro', Conrado Neves Sathler², \\ Catia Paranhos Martins ${ }^{2}$ \\ ' Programa de Pós-graduação Stricto sensu Ensino em Saúde, Mestrado Profissional, Universidade Estadual de \\ Mato Grosso do Sul, Dourados (MS), Brasil. \\ ${ }^{2}$ Programa de Residência Multiprofissional em Saúde, Hospital Universitário, Universidade Federal da Grande \\ Dourados, Dourados (MS), Brasil.
}

\section{RESUMO}

Objetivo: A experiência docente é apresentada neste artigo através da descrição da trajetória de formação de profissionais de saúde em um programa de Residência Multiprofissional em Saúde (RMS), que se caracteriza pelo ensino em serviço, na modalidade especialização lato sensu, orientada pelos princípios e diretrizes do Sistema Único de Saúde (SUS). O RMS do Hospital Universitário da Grande Dourados - Mato Grosso do Sul - atua na saúde indígena, considerando as características regionais. A cidade de Dourados tem a segunda maior população indígena do Brasil. Durante a formação, os alunos (trabalhadores de saúde) são capacitados para conhecer a diversidade e a complexidade do contexto social, histórico e cultural dos povos indígenas. Método: Pesquisa qualitativa de revisão narrativa que busca refletir sobre a prática docente e a formação de trabalhadores em saúde indígena. Resultados: A RMS é identificada como um lugar privilegiado para expandir a compreensão da saúde e da doença dos povos indígenas. Há muitos desafios, por exemplo, implementar e fortalecer as políticas públicas, avançar nas ações em rede e intervir em aspectos sociais. Conclusões: Podemos perceber riquezas como o trabalho pautado nos princípios e diretrizes do SUS; a clínica ampliada; os vínculos entre trabalhadores de saúde e as comunidades indígenas; o acolhimento dos profissionais nos diversos espaços onde os residentes atuam. A rede de saúde é também rede de vínculos, de produção de vida. Construímos uma formação em saúde que mobiliza os estudantes e docentes para ações além das prescrições no currículo.

Recebido: Maio 31, 2016 Aceito: Nov. 06, 2016

\section{COMO CITAR ESTE ARTIGO}

Abilio ES, Cordeiro MJJA, Sathler CN, Martins CP. Experiência docente em saúde indígena: reflexões e movimentos. Interdisciplinary Journal of Health Education. 2016 Ago-Dez;1(2):100-105 http://dx.doi.org/10.4322/ijhe.2016.018

\section{CORRESPONDÊNCIA}

Elenita Sureke Abilio

Programa de Pós-graduação Stricto sensu Ensino em Saúde, Mestrado Profissional, Universidade Estadual de Mato Grosso do Sul

Rua Odalíria Olegário de Oliveira, 1845 CEP 79823-840, Dourados (MS), Brasil lesurekeabilio@gmail.com

\section{FONTE DE FINANCIAMENTO}

Estudo autofinanciado.

\section{CONFLITO DE INTERESSE}

Os autores declararam não

haver conflitos de interesse.

O estudo foi realizado na Biblioteca da Universidade Federal da Grande Dourados, Dourados (MS), Brasil.

Todos os autores leram e aprovam a versão final submetida ao Interdisciplinary Journal of Health Education (IJHE).
PALAVRAs-Chave: Saúde de populações indígenas. Educação em saúde. Psicologia.

\section{ABSTRACT}

Objective: The teaching experience is presented in this article through the description of the training trajectory for health professionals in a Multiprofessional Health Residency (RMS) program, characterized by in-service teaching, in the lato sensu specialization modality, guided by the principles and guidelines of the Unified Health System (UHS). The RMS of the University Hospital of Grande Dourados - Mato Grosso do Sul - operates in indigenous health, considering the regional characteristics. The city of Dourados has the second largest indigenous population in Brazil. During their training, students (health workers) are trained to understand the diversity and complexity of the social, historical and cultural context of indigenous people. Method: Qualitative research of narrative review that seeks to reflect on the teaching practice and the training of indigenous health workers. Results: The RMS is identified as a privileged place to expand the understanding of the health and disease of indigenous people. There are many challenges, for example, to implement and to strengthen public policies, to advance networking as well as to intervene in social aspects. Conclusions: It was noticed that the riches such as the work based on the principles and guidelines of UHS; the expanded clinic; the linkages between health workers and indigenous communities and the reception of professionals in the various spaces where residents work. The health network is also a network of links, i.e., of life production. It is built a health education that mobilizes students and teachers for actions beyond those prescribed in the curriculum.

KEYWORDS: Health of indigenous people. Health education. Psychology. 


\section{Introdução}

É necessário refletir sobre a docência em saúde como um desafio para as ações pedagógicas em decorrência de novas exigências e um novo perfil docente, o que demanda uma análise crítico-reflexiva das ações educacionais, principalmente aquelas atreladas à área da saúde. Este artigo traz em seu contexto a atuação dos autores no campo de ensino como preceptores e gestores de um programa de pós-graduação na modalidade residência que contempla três profissões da saúde, sendo uma delas a Psicologia.

Assim, o destaque é para a profissão de Psicologia, por ser a área profissional de três dos autores, que marca a experiência docente em um momento singular de intensificação dos estudos para atender as demandas pedagógicas, subjetivas, discursivas e analíticas da atenção à saúde indígena com um olhar cuidadoso para o ensino em saúde ${ }^{1}$. O aguçar pelas teorias educacionais vinculadas à saúde através da Política Nacional de Educação Permanente em Saúde (EPS) ${ }^{2}$ trouxe as oportunidades de qualificação profissional e o investimento do trabalho na área de formação e pesquisa e ainda marcaria não apenas uma prática de ensino e aprendizagem, mas a constituição de fato de um processo político de educação na saúde ${ }^{3}$.

Dourados é a segunda maior cidade do estado do Mato Grosso do Sul e contempla a segunda maior população indígena do Brasil com predominância das etnias Guarani, Kaiowá e Terena. Neste município ${ }^{4}$ as aldeias são localizadas próximas do centro urbano e são conhecidas por serem as mais populosas do país, possuírem índice elevado de suicídio e por conterem a maior população carcerária indígena.

As características tão marcantes desta população são objeto de estudo de vários cursos nas instituições de ensino superior. Na Universidade Federal da Grande Dourados (UFGD) destacam-se os cursos de Antropologia e Psicologia, interligados ao Programa de Residência no seu currículo, e as contribuições da Faculdade Intercultural Indígena, criada em 2012, que fortaleceram as ações educativas para esta população.

A Residência Multiprofissional em Saúde (RMS) se caracteriza pelo ensino em serviço na modalidade especialização lato sensu, regida pela promulgação da Lei $n^{\circ} 11.129$ de 2005 e orientada pelos princípios e diretrizes do Sistema Único de Saúde (SUS) 5 .

A proposta do Programa no Hospital Universitário da Universidade Federal da Grande Dourados tem em uma de suas linhas de atuação a Atenção à Saúde Indígena, considerando as características loco regionais tão marcantes no Estado, e contempla três profissões da saúde: Enfermagem, Nutrição e Psicologia.

Considerando as questões sanitárias da região, discutir o olhar ampliado em saúde ${ }^{6}$, levando em conta as questões indígenas abriu-se um novo campo de atuação, limitado pelo pouco conhecimento e abertura curricular para estes novos desafios. Foi necessário muito mais investimento do que os prescritos formalmente no projeto pedagógico, além de investimento cognitivo, temporal e didático.

Analisar o território de saúde e as características loco regionais, bem como a oferta de trabalho em saúde no sistema público através dos concursos e processos seletivos para os profissionais psicólogos, demandava um investimento teórico e pratico além da prescrição na matriz curricular e no projeto pedagógico do curso. Algumas demandas trouxeram um aguçar pelas teorias do currículo, marcando um encontro potente entre a educação e a saúde ${ }^{7}$.

Portanto, o objetivo deste artigo é descrever o investimento no aperfeiçoamento docente a partir da prática do trabalho de formação em saúde, evidenciando as experiências exitosas neste percurso e que fazem parte da qualificação de profissionais de saúde para atender as demandas sociais e sanitárias da saúde indígena na região. 


\section{Métodos}

O método de pesquisa é a pesquisa qualitativa utilizando-se como procedimento de estudo a revisão narrativa que busca analisar a prática docente por meio da crítica ao processo de qualificação e o investimento pedagógico necessário para a formação de residentes multiprofissionais na saúde indígena.

Para o levantamento bibliográfico, optou-se pela busca de artigos em periódicos nacionais, disponíveis nas bases de dados pertencentes à Biblioteca Virtual em Saúde (BVS) e Scientific Electronic Library Online (SCIELO). Foram utilizados os seguintes descritores: saúde indígena, ensino em saúde e residência multiprofissional. Como critérios de inclusão, os artigos deveriam preencher as seguintes condições: apresentar experiências de ensino em saúde com foco na Educação Permanente em Saúde e serem artigos publicados em português. Foram excluídos artigos de revisão bibliográfica. A análise foi realizada considerando informações específicas de cada artigo.

A construção desta pesquisa realizou-se a partir de procedimentos distintos, porém interligados. A pesquisa bibliográfica, envolvendo estudos acerca da formação na modalidade residência e os desafios para implementação desta modalidade de pós-graduação, reconhecendo a importância do investimento docente na formação dos residentes e o impacto social para implementação e efetividade das políticas de saúde.

\section{Resultados e discussões}

A natureza do Programa de RMS é a formação multiprofissional que abre o campo de intervenção para a dimensão ampliada, envolvendo as questões socioculturais e políticas, sendo esta uma nova demanda a ser investida no currículo para atender as habilidades e competências exigidas no Projeto Pedagógico ${ }^{8}$, norteada pela Política Nacional de Educação Permanente em Saúde ${ }^{2}$.

Desde a sua implantação em 2010, o Programa tem mostrado relevante impacto social a partir da inserção dos residentes tanto na rede hospitalar quanto na rede da Atenção Básica, que prevê a participação ativa dos residentes, sob a supervisão de profissionais da equipe de saúde e docentes da universidade credenciados no Programa de Residência Multiprofissional.

O interesse de acadêmicos e profissionais por esta especialização, a necessidade de qualificação na área da saúde e também a busca de incentivo ao desenvolvimento científico, tornam a implantação do Programa de Residência uma estratégia importante para a Atenção à Saúde na região da Grande Dourados e para o Estado do Mato Grosso do Sul.

Com um olhar de análise da caminhada deste processo de formação dos residentes, entendemos que a compreensão de tal experiência é reconhecida como transformadora, dentro da sua diversidade cultural, de tempo, espaço, ambiência, entre outros aspectos. Isso nos ajuda a entender a diferença e o hibridismo como riqueza, e não como empecilho a uma prática de saúde e sentido9.

Alguns desafios foram colocados nesse percurso, como as políticas públicas insuficientes, os avanços nas grades de ensino que tratem das questões indígenas, por exemplo, políticas públicas de saúde indígena/indigenista e o impacto dos determinantes sociais no trabalho das equipes de saúde ${ }^{10}$.

O encontro com os profissionais de saúde e a comunidade, no território em questão, possibilitou aos residentes compreender saberes e práticas e sua relação com o Sistema Único de Saúde. Além disso, proporciona visualizar as reais dimensões e importância do trabalho em rede, considerando saúde como um conceito ampliado que produz $v_{\text {vida }}{ }^{11}$. Os vínculos entre trabalhadores de saúde, em especial as Agentes Indígenas de Saúde e a comunidade indígena, o acolhimento dos profissionais dos diversos 
espaços onde os residentes caminharam e a rede de saúde como rede de vínculos e de produção de vida são as maiores riquezas desse aprendizado.

No Projeto Pedagógico ${ }^{8}$ está preconizado que ao final da formação, o residente de psicologia na área de concentração Atenção à Saúde Indígena deverá ter desenvolvido algumas habilidades necessárias para a atuação profissional, como: o reconhecimento da diversidade e complexidade do contexto sócio-histórico-cultural dos povos indígenas no Brasil; a atuação em equipe de forma interdisciplinar no âmbito da Atenção à Saúde Indígena; o planejamento de intervenções a indivíduos; família e coletividade na Atenção à Saúde Indígena; a promoção em educação em Saúde Indígena; a atuação na rede de serviços de e o desenvolvimento da pesquisa gerando conhecimentos que contribuam para o aprimoramento das práticas em Saúde Indígena.

A saúde indigenista constitui os serviços e ações formuladas e implementadas com base nas concepções do processo saúde-doença da sociedade ocidental e são direcionados aos índios. Saúde indígena refere-se aos saberes curativos dos povos indígenas, de acordo com as suas próprias concepções ${ }^{12}$.

A experiência no Programa de Residência é reconhecida como um lugar privilegiado para entendimento de várias questões que tocam a temática indígena, sendo questões culturais, a construção de subjetividades, a constituição histórica, a problemática da demarcação de terras e, ainda, as intervenções e ações em saúde ${ }^{13}$.

Sair das paredes da instituição hospitalar e caminhar pela rede de saúde, proporciona o encontro com outras áreas do conhecimento como a Antropologia da Saúde, contribuindo para a aproximação com culturas diversas, como a das populações indígenas, representadas em nosso contexto, sobretudo, pelas etnias Guarani, Kaiowá. Alguns desafios são colocados nesse percurso, como as políticas públicas insuficientes, avanços nas grades de ensino que tratem das questões indígenas e o impacto dos determinantes sociais no trabalho das equipes de saúde.

A formação acadêmica nas áreas de saúde tende a ser fragmentado, o que dificulta o trabalho multiprofissional. Ainda, poucos estão familiarizados com um conceito de saúde ampliado que nos oferece subsídios, como instrumento teórico de nova ordem, de perspectiva transdisciplinar e totalizante ${ }^{14}$.

Durante a formação, os residentes são capacitados a reconhecer a diversidade e a complexidade do contexto sócio-histórico-cultural dos povos indígenas, atuar de forma multiprofissional e demais intervenções em saúde.

As vivências do trabalho são iniciadas no Hospital Universitário como principal cenário de prática no Projeto Pedagógico. Apesar de ricas em seu contexto, aliadas a formação acadêmica, dificultam a compreensão do trabalho em rede, já que é fundamental considerar o Subsistema de Saúde Indígena, na construção da atenção integral.

A necessidade da caminhada em toda a Rede de Atenção à Saúde do município, propiciada em um dos poucos estágios da ênfase, oportuniza aos residentes conhecer a Atenção Básica e a Atenção Especializada, bem como Atenção Básica na Saúde Indígena, o que colabora para a construção de um inovador e desafiante processo de trabalho em saúde. Com isso, possibilita aos residentes conhecerem a rede de serviços de saúde, de suporte social, de gestão e de controle social.

Ao relacionar-se com comunidades indígenas, o sistema de saúde se depara com a dimensão intercultural, o que remete diretamente à discussão sobre a adequação ética e cultural das práticas sanitárias ocidentais e sua relação com os sistemas indígenas de saúde. Há que indagar-se, [...], sobre a potencialidade desse encontro em produzir afetações que interroguem a biomedicina e as formas de conceber e organizar os serviços de maneira mais ampla ${ }^{19}$ (p. 871).

É evidenciado que o idioma dos usuários e o vocabulário técnico utilizado pela biomedicina não atendem as necessidades de saúde em sua complexidade ${ }^{16}$. Na nossa experiência, a linguagem, se por um lado representa para alguns sujeitos envolvidos 
impedimento de contato com o outro, para muitos, representa ao contrário, a chance de encontro com o outro. Seja no desejo e empenho por conhecer outra língua, aproximar-se de outra cultura ou até mesmo no intento de desenvolver junto ao usuário outras formas de comunicação, para além da comunicação verbal.

Baseado nas pesquisas sobre o quadrilátero da formação ${ }^{17}$ reafirmamos a importância da formação em saúde para atender aos preceitos do SUS, marcadas pelos encontros que fortalecem o trabalho em saúde, como o encontro da Saúde com a Educação, do Trabalho com a Gestão na condução da formação dos profissionais de saúde.

Consta no Projeto Pedagógico uma disciplina denominada Tópicos Especiais. Esta disciplina é conduzida para cada uma das áreas de formação separadamente com o objetivo de discutir temas diversos relacionados ao núcleo da profissão que são selecionados pelos tutores, preceptores e residentes ${ }^{2}$. Alguns temas são pré-selecionados pelos tutores e preceptores antecipadamente, considerando a complexidade das ações multiprofissionais e institucionais vivenciadas no histórico do Programa. Para a psicologia destacamos a importância desta disciplina para as conexões com a diversidade sócio-cultural na região de saúde, as questões antropológicas e a riqueza da atenção em rede para a resolubilidade dos serviços do SUS. Dentro da carga horária que é destinada ao núcleo profissional, há alguns tópicos que devem ser ministrados no campo das profissões, e há os temas de Políticas Públicas, Ética e Bioética que são ministrados para as três profissões em conjunto.

Dentro do currículo, este foi o recurso utilizado para aprimorar as discussões inerentes a cada uma das profissões, trazendo embasamento teórico para os tópicos relacionados às questões do conceito ampliado de saúde, as questões indígenas, além do investimento dos docentes em projetos de pesquisa e extensão para o aprimoramento da formação dos residentes.

A caminhada durante a RMS permite a aproximação dos residentes da multiplicidade territorial e das fronteiras existentes nesse $\operatorname{contexto}^{18}$, onde sujeitos distintos carregam noções distintas, ficando clara a diferença entre indígenas e não indígenas, e como isso influencia em seus modos de ser e agir.

\section{Conclusão}

No campo do trabalho, os residentes formados no programa tem se destacado assumindo funções estratégicas nas áreas de atenção a saúde, demonstrando um diferencial na formação quando se inserem nas discussões e ações de controle social. Ainda consideramos a formação como estratégia para a qualificação dos residentes para atuação no eixo de formação ${ }^{7}$, vinculados em espaços de ensino a partir das experiências no programa, tanto como docentes, como no ingresso na pós-graduação stricto senso.

O processo de implantação do Programa de Residência Multiprofissional em Saúde (RMS) do HU/UFGD necessita de discussões pedagógicas nos novos cenários de prática que configuram a rede de atenção à saúde e remetem o profissional não apenas a formação generalista, dentro do seu núcleo de formação. Um núcleo indica junção de saberes e de práticas, sem, contudo, indicar um rompimento radical com a dinâmica do campo, exigindo dos residentes uma postura crítico-reflexiva que compreenda, também, as relações humanas como parte do ensino ${ }^{17}$.

Com um olhar para o processo de formação entendemos que a compreensão de tal experiência é reconhecida como transformadora, dentro da sua diversidade cultural, de tempo, espaço, ambiência, etc. Isso nos ajuda a entender a diferença e o hibridismo como riqueza, e não como empecilho a uma prática de saúde ${ }^{10}$.

Embora os desafios sejam muitos, os vínculos entre trabalhadores de saúde, a comunidade, o acolhimento dos profissionais/pessoas dos diversos espaços onde os residentes caminharam fazem-nos apostar na construção da rede de saúde como uma rede de vínculos e produção de vida. 
Os resultados do trabalho apontam que as atividades de formação se desenvolvem no cenário hospitalar, perpassando por várias unidades de atendimento, contemplando a rede intra-hospitalar e a rede extra-hospitalar, tendo como demais cenários de prática, unidades básicas de saúde e da atenção especializada, as aldeias indígenas de Dourados, o Hospital indígena e os cenários de atuação da Secretaria Especial de Saúde Indígena que compõem a rede de saúde, convocando mobilização dos docentes para ações além das prescritas no currículo formal.

\section{Referências}

1. Brasil. Resolução CNE/CES n 5 , de 15 de março de 2011. Institui as Diretrizes Curriculares Nacionais para os cursos de graduação em Psicologia. Diário Oficial da União, Brasília, DF, 7 fev. 2011.

2. Brasil. Portaria GM/MS n ${ }^{\circ}$ 1.996/07, de 20 de agosto de 2007. Dispõe sobre as diretrizes para a implementação da Política Nacional de Educação Permanente em Saúde. Diário Oficial da União, Brasília, DF, 1 nov. 2007.

3. Ceccim RB, Ferla AA. Educação e saúde: ensino e cidadania como travessia de fronteiras. Trab. Educ. Saúde. 2008;6(3):44356. http://dx.doi.org/10.1590/S1981-77462008000300003.

4. Martins CP. Considerações sobre o processo de humanização no HU de Dourados - MS. Brasília (DF): Ministério da Saúde; 2011.

5. Brasil. Portaria Interministerial $n^{\circ} 45$, de 12 de janeiro de 2007. Organiza e disciplina no âmbito dos Ministérios da Saúde e Educação a criação da Comissão Nacional de Residências Multiprofissionais em Saúde. Diário Oficial da União, Brasília, DF, 15 jan. 2007.

6. Merhy EE, Feuerwerker LCM. Novo olhar sobre as tecnologias de saúde: uma necessidade contemporânea. In: Mandarino ACS, Gomberg E, organizadores. Leituras de novas tecnologias e saúde. São Cristóvão: Editora UFS; 2009. p. 29-74.

7. Ceccim RB. Educação permanente em saúde: desafio ambicioso e necessário. Interface. 2005;9(16):161-68. http://dx.doi. org/10.1590/S1414-32832005000100013.

8. Universidade Federal da Grande Dourados (UFGD). Hospital Universitário. Projeto Pedagógico do HU-UFGD: define diretrizes pedagógicas do Programa de Residência Multiprofissional em Saúde do Hospital Universitário da Universidade Federal da Grande Dourados. Dourados: HU-UFGD; 2009.

9. Diehl E, Pellegrini MA. Saúde e povos indígenas no Brasil: o desafio da formação e educação permanente de trabalhadores para atuação em contextos interculturais. Cad Saude Publica. 2014;30(4):867-74. http://dx.doi.org/10.1590/0102-311X00030014.

10. Luciano GS. O índio brasileiro: o que você precisa saber sobre os povos indígenas no Brasil de hoje. Brasília (DF): MEC/ SECAD; LACED/Museu Nacional; 2006.

11. Merhy EE. Saúde: a cartografia do trabalho vivo. 3. ed. São Paulo: Editora Hucitec; 2002.

12. Cruz KR, Coelho EMB. A saúde indigenista e os desafios da particip(ação) indígena. Saude Soc. 2012;21(1, Supl 1):185-98. http://dx.doi.org/10.1590/S0104-12902012000500016.

13. Turdera GB. O contexto da saúde e doença indígena: a experiência da residência multiprofissional de Dourados - MS. In: VII Congresso internacional de Psicopatologia Fundamental; XII Congresso Brasileiro de Psicopatologia Fundamental. O Pathos nas práticas de Saúde: representações psicopatológicas, antropológicas e institucionais; 2014 Set 4-7; Belo Horizonte. Belo Horizonte; 2014. p. 1-7.

14. Batistella C. Abordagens contemporâneas do conceito de saúde. In: Fonseca AF, organizador. O território e o processo saúde-doença. Rio de Janeiro: EPSJV/Fiocruz; 2007. p. 51-86.

15. Santos EF, Daniel L. Educação escolar indígena e suas interfaces com o campo da saúde. In: Garnelo L, Pontes AL, organizadores. Saúde indígena: uma introdução ao tema. Brasília: Secretaria de Educação Continuada, Alfabetização, Diversidade e Inclusão; 2012. p. 242-63.

16. Pereira PPG. Limites, traduções e afetos: profissionais de saúde em contextos indígenas. Mana. 2012;18(3):511-38. http:// dx.doi.org/10.1590/S0104-93132012000300004.

17. Ceccim RB, Feuerwerker LCM. O quadrilátero da formação para a área da saúde: ensino, gestão, atenção e controle social. Physis: Rev. Saúde Col. 2004;14(1):41-65.

18. Silva CDC. Saúde e política: uma etnografia dos profissionais da saúde indígena [tese]. Brasília (DF): Universidade de Brasília; 2010.

19. Campos GWS. Saúde pública e saúde coletiva: campo e núcleo de saberes e práticas. Cien Saude Colet. 2000;5(2):219-30. http://dx.doi.org/10.1590/S1413-81232000000200002.

\section{Contribuição dos autores}

Os autores Elenita Sureke Abilio, Maria José de Jesus Alves Cordeiro, Conrado Neves Sathler, Catia Paranhos Martins, tiveram participação suficiente no trabalho para assumir a responsabilidade pelo conteúdo integral, concepção e planejamento do projeto de pesquisa; obtenção ou análise e interpretação dos dados, redação e revisão crítica do texto/conteúdo. 